\title{
La mesure des progrès lexicaux en FL2 avancé
}

\author{
Alain Thomas \\ University of Guelph \\ thomas@uoguelph.ca
}

\section{Introduction}

Bien que les échanges d'étudiants entre universités de langues différentes existent depuis plus d'un siècle, ce n'est que depuis une douzaine d'années qu'on s'est mis à évaluer sérieusement les avantages et inconvénients de ce genre d'expérience, dépassant ainsi la simple constatation habituelle d'un certain 'progrès' en langue seconde (dorénavant 'L2'). Comme le faisait remarquer Barbara Freed dans un premier survol de la question, "no study has yet described a range of linguistic variables (phonologic, syntactic and/or semantic), sociolinguistic and discourse features, that may be influenced as a result of a study abroad experience" (1995:16). Même récemment, Joseph Collentine constatait encore une certaine paucité d'études comparant les programmes réguliers et les études linguistiques à l'étranger (2004: 228).

Afin de tenter de combler en partie cette lacune, du moins pour le français, nous avons entrepris une recherche empirique portant sur les progrès linguistiques accomplis en français langue seconde par deux groupes d'étudiants avancés ayant passé leur troisième année universitaire en France (groupe expérimental) ou dans le sud de l'Ontario (groupe témoin). Le présent article se limitera à l'analyse qualitative et quantitative des progrès accomplis par ces étudiants sur le plan lexical, dont l'importance est de plus en plus reconnue dans la recherche en langue seconde. On pourra ainsi vérifier pour le FL2 si le séjour en France favorise effectivement l'acquisition lexicale et, si oui, jusqu'à quel point et dans quels domaines. La méthodologie utilisée tentera de tenir compte des principaux reproches faits aux études antérieures pertinentes, comme on le verra ci-dessous.

\section{Recherches antérieures}

La plupart des recherches sur la valeur linguistique des séjours à l'étranger sont concentrées sur la grammaire (maigres progrès) et sur la facilité et la rapidité avec laquelle les sujets s'expriment oralement (gros progrès). Bien peu sont consacrées spécifiquement au vocabulaire et elles sont toutes récentes (moins de dix ans; cf. Churchill et Dufon, 2006, pour une vue d'ensemble). La rareté relative des études lexicales dans l'acquisition des langues secondes tient probablement à la difficulté de quantifier les phénomènes observés et de les analyser systématiquement en termes de règles, comme dans les autres domaines de la linguistique (Meara, 1984: 230). Alors que les phonéticiens ont beau jeu d'isoler et d'analyser l'occurrence répétée de tel ou tel phonème, et que les grammairiens peuvent facilement étudier par exemple les terminaisons verbales, les lexicologues s'attaquent à un système ouvert, où les récurrences sont trop rares pour justifier des analyses statistiques. Pourtant, les erreurs lexicales sont considérées bien plus graves que celles de grammaire (Meara, 1984: 229; Laufer 1986: 69) et c'est bien dans le lexique qu'on trouve l'essentiel des significations, comme on peut le voir dans les textos des jeunes d'aujourd'hui ou dans les télégrammes d'autrefois. Par exemple, la séquence aéroport - 15 heures - samedi - Suzanne - arrive reste parfaitement claire, malgré ses carences évidentes en morpho-syntaxe. Finalement, avec un bon vocabulaire, on arrive toujours à se débrouiller en L2 et on ne saurait parler couramment sans une solide base lexicale (Laufer, 1986: 73).

Les résultats de ces recherches signalent généralement des progrès lexicaux importants pendant l'année à l'étranger, du moins aux niveaux debutant / intermédiaire. C'est surtout le cas pour Milton et Meara (1995) qui, à partir d'un test informatisé à choix binaire et d'un questionnaire, arrivent à la conclusion qu'on peut 
améliorer sa connaissance du vocabulaire anglais de 23\% pendant un séjour de 6 mois en Angleterre, c'est-àdire au moins 4 fois plus que si on était resté chez soi pour étudier cette langue. Ces gains impressionnants sont indépendants de la langue maternelle (L1) des sujets ou du temps passé aux études, mais ils semblent

reliés à une attitude positive et à un contact intense avec les familles d'accueil, et ils sont particulièrement évidents chez les sujets les plus faibles en anglais au début du séjour.

Au niveau avancé, les étudiants, qui ont déjà maîtrisé l'essentiel de la grammaire et de la phonologie de leur L2, se distinguent surtout par l'étendue et la précision de leur vocabulaire (Laniel, 2005: 76). Leurs productions se dégagent progressivement du lexique élémentaire et répétitif des débuts au profit d'un vocabulaire plus nuancé, mais elles restent souvent caractérisées par des interférences de leur L1 (forme ou sens) et par l'absence relative de mots non-standard (Dewaele et Pavlenko, 2002), de métaphores et d'expressions idiomatiques (Cobb, 2003).

Les études antérieures sur le lexique en L2 sont souvent caractérisées par des limitations qui les empêchent d'arriver à des conclusions claires et solides (voir à sujet Freed, 1995, et Collentine, 2004). D'abord, faute de moyens, on n'a pas vraiment étudié la production spontanée des sujets, préférant se concentrer sur des tests spécifiques (choix multiple, mots à compléter, par exemple) mesurant surtout des connaissances lexicales passives. Les analyses ont par ailleurs été faites le plus souvent sur des échantillons réduits, à faible valeur statistique, et parfois à l'aide de tests trop faciles masquant les progrès à cause de l'effet de 'plafond'. Enfin, on a rarement considéré les contextes d'apprentissage: à quoi bon établir que les étudiants ont fait des progrès pendant leur année à l'étranger si on ne compare pas ces progrès à ceux réalisés par des sujets semblables qui sont restés au pays pendant la même période?

De nouveaux outils électroniques, cependant, sont maintenant disponibles pour faciliter la quantification des données et, comme l'ont noté divers spécialistes, le domaine de l'acquisition lexicale en L2 suscite un intérêt grandissant parmi les chercheurs. Le plus utile de ces outils, le logiciel Vocabprofile de Laufer et Nation (1995) a permis de mesurer la richesse lexicale de textes écrits en anglais, en calculant la proportion des mots les plus rares, c'est-à-dire ceux qui dépassent le 2000e rang dans les listes de fréquence. Il a été adapté pour le français par Jones (2001), puis validé et utilisé d'abord par Goodfellow et Lamy (2002) sur un corpus de français L2 faible, puis par Ovtcharov et al. (2006) sur un corpus de français L2 avancé, fourni par des fonctionnaires Anglophones canadiens. Ces deux études concluent qu'on peut évaluer la richesse lexicale des textes français dès le $1000 \mathrm{e}$ rang de fréquence, contrairement à l'anglais, pour lequel il vaut mieux commencer au seuil du 2000e rang. Cobb et Horst (2004) expliquent cette divergence en termes d'importance comparée de la 2e zone de fréquence (1000e - 2000e rang), qui est beaucoup plus utile en français qu'en anglais pour exprimer les nuances lexicales.

Comme ces deux études, la nôtre utilise le logiciel Vocabprofil, qui est d'une précision et d'une richesse étonnantes, et qui a déjà fait ses preuves. Cela devrait nous mener à des comparaisons intéressantes avec les résultats d'Ovtcharov et al, puisqu'il s'agit encore du français L2 avancé, en contexte canadien. Nous allons cependant plus loin que nos prédécesseurs en doublant la taille du corpus, en ajoutant une dimension intraindividuelle aux comparaisons inter-individuelles pour mieux cerner la notion de 'progrès', et en complétant l'étude par une dimension qualitative, qui nous paraît essentielle à une meilleure interprétation des statistiques brutes offertes par l'analyse quantitative. Comme le soulignait autrefois le grand linguiste et statisticien Charles Muller, "il va de soi que rien de solide ne peut être dit de la richesse lexicale relative d'un texte sans un dépouillement lexical complet” (1979:307).

\section{Protocole d'enquête}

Plusieurs universités sud-ontariennes dotées d'un programme de français de 3e année en France ont été choisies comme source de données. Grâce à l'aimable collaboration des administrateurs responsables de ces 
programmes, on a pu tester presque une centaine d'étudiants de $3 \mathrm{e}$ année. Les participants retenus pour l'enquête ont été évalués au moyen d'un test sommatif oral, administré au début (pré-test) et à la fin (posttest) de l'année académique dans des conditions quasi-identiques. L'enquête tentait d'autre part, outre la

dimension expérimentale mentionnée en introduction, de brosser un tableau des compétences et des faiblesses de nos étudiants avancés de français, à des fins correctives et pédagogiques.

La base de l'évaluation est un test de 52 minutes, entièrement pré-enregistré (même les pauses), qui pouvait donc être administré par toute personne - y compris un-e anglophone unilingue - disposant du matériel nécessaire. Le remplacement occasionnel de l'enquêteur par un collègue enseignant dans ce rôle et le passage d'un laboratoire à un autre - ou même parfois au magnétophone individuel - ne devraient donc avoir aucune incidence sensible sur les résultats. Par ailleurs, tous les participants savaient que le test était confidentiel et entièrement étranger à leur dossier universitaire. Ils n'étaient pas obligés de participer, mais l'ont généralement fait de bon gré, par altruisme ou par intérêt personnel, pour recevoir un résumé de leur performance individuelle, qui leur a effectivement été envoyé l'année suivante. Ceci dit, il faut reconnaître que certains facteurs possibles de variation, indépendants de notre volonté, ont pu affecter la performance des étudiants. Est-on aussi motivé en fin d'après-midi qu'à $9 \mathrm{~h}$ du matin (les bâillements d'une certaine participante en disent long à ce sujet...)? En fin d'année scolaire qu'au début? En enregistrement individuel qu'en laboratoire de langues? En présence d'un professeur habituel qu'en présence d'un inconnu? Par ailleurs, la notion d'"année universitaire" est nécessairement plus flexible ici (entre 7 et 9 mois) que dans nos annuaires officiels. Notre corpus comporte donc plusieurs petites faiblesses pratiquement inévitables quand on travaille avec des êtres humains et dans les conditions d'économie de temps et de moyens qui étaient forcément les nôtres. Nous croyons cependant qu'il n'en reste pas moins valable dans son ensemble et que ses faiblesses qualitatives sont partiellement compensées par sa dimension quantitative, qui dépasse largement la norme habituelle dans ce genre d'enquête.

Après avoir éliminé les enregistrements inutilisables, pour raisons techniques, test incomplet ou surtout absence de pré-test ou de post-test, il nous est resté 87 sujets, tous étudiants de $3 \mathrm{e}$ année inscrits au moins à 4 cours semestriels de français pendant l'année de l'enquête. Ils étaient répartis entre les 7 universités participantes de la manière suivante:

\begin{tabular}{|l|l|l|l|l|l|l|l|}
\hline Western & Guelph & Windsor & Laurier & Waterloo & Toronto & Trent & TOTAL \\
\hline 12 & 29 & 10 & 5 & 20 & 4 & 7 & 87 \\
\hline
\end{tabular}

A partir de leur expérience antérieure du français, on a pu les répartir initialement en trois groupes distincts: "français cadre" (40), “immersion" (41) et "francophone" (6). L'expérience a montré cependant que ces divisions étaient plus théoriques que réelles et qu'elles avaient souvent été effacées, du moins d'après les résultats de notre test, avant l'arrivée en 3e année d'université. Les 6 "francophones" en particulier, tous issus d'un milieu franco-ontarien minoritaire, n'ont pas nécessairement commencé plus haut que les autres, ni fait moins de progrès pendant l'année (à cause de l'effet de 'plafond' qui interdit les grands progrès à ceux qui sont déjà très près du but). Ils ont finalement été maintenus dans l'enquête, car ils s'inscrivaient comme tout le monde dans un continuum de compétences allant d' 'intermédiaire' à 'très avancé' et ils appartenaient en tout cas à ce groupe d'étudiants avancés dont on se proposait de décrire les compétences. Notons par ailleurs que l'enquête est basée essentiellement sur la mesure de progrès réalisés en une année et non sur des compétences acquises antérieurement. Pour ces raisons donc, la dimension 'compétence antérieure' n'a pas été retenue dans l'analyse quantitative. L'origine linguistique des sujets, qui fait évidemment partie de cette dimension et qui n'a pas été retenue pour les francophones, ne l'a pas été non plus pour les quelques italophones et sinophones du corpus. A toutes fins pratiques, on a pris des étudiants situés au départ assez 
haut sur une échelle de compétence en français, pour quelque raison que ce soit (mais suffisamment loin de la perfection pour permettre des progrès), et on a examiné les progrès accomplis en une année universitaire. Notons enfin que, même si la dimension 'langue maternelle' a pu affecter les résultats, elle a été partiellement

gommée statistiquement par le fait que les non-anglophones constituaient en tout et pour tout moins de $15 \%$ de notre corpus.

Pour répondre à la question principale de cette recherche (cf. Freed, ci-dessus), on a divisé les sujets en fonction de la nature et de l'intensité de leur expérience linguistique pendant l'année de l'enquête. Alors que les sujets restés en Ontario (groupe "témoin" de 39 étudiants) ont suivi entre 4 et 6 cours semestriels de français en milieu anglophone, les sujets partis en France (Nice ou Nantes, groupe "expérimental" de 48 étudiants) ont suivi entre 8 et 10 cours en français, et surtout en milieu francophone. On s'attend évidemment à ce que ces derniers fassent plus de progrès que leurs camarades restés au pays. La question étudiée ici est de documenter la nature qualitative et quantitative de ces progrès.

La partie du test utile à l'analyse lexicale du corpus comprend une épreuve d'expression orale semi-directe d'une durée de 13 minutes et basée sur trois épreuves consécutives enregistrées individuellement ou collectivement dans un laboratoire de langues:

- Description d'une scène de rue riche en personnages et anomalies, à partir d'un dessin de couverture du magazine New Yorker.

- Description favorable de l'université de l'étudiant, à des fins publicitaires en milieu francophone.

- Autobiographie, exprimée au passé.

On voit qu'il s'agit d'exercices variés, réalistes et aussi peu intimidants que possible, puisqu'ils ne requièrent aucune connaissance particulière et qu'ils sont limités à l'univers familier des étudiants. Nous croyons, avec Tréville et Duquette (1996:159), que les tâches proposées pour ce discours monologual sont appropriées à l'évaluation du bagage lexical des sujets et qu'elles sont bien plus prometteuses, en tout cas, que les listes de mots décontextualisés utilisés dans certains types de recherches. Comme on a permis aux sujets de choisir eux-mêmes le meilleur moment pour passer d'une épreuve à l'autre - évitant ainsi des moments de silence embarrassants à ceux qui n'étaient pas inspirés par le dessin - la quantité de texte produite dans chaque cas peut varier considérablement, souvent au détriment de la dernière épreuve. Bien que cette décision ait réduit la validité statistique de certaines mesures au niveau individuel, elle n'a pas eu de conséquences graves au niveau collectif.

La production orale des sujets a d'abord été transcrite aussi fidèlement que possible en orthographe conventionnelle (avec hésitations, reprises, etc.) pour l'analyse qualitative, puis adaptée aux exigences du logiciel Vocabprofil pour l'analyse quantitative. Vocabprofil est une adaptation pour le français du logiciel Lexical Frequency Profile (Laufer et Nation, 1995; disponible sur www.lextutor.ca), qui mesure la richesse lexicale d'un texte en comparant ce dernier (mots, vocables et familles) à des listes de fréquence pré-établies. Ces listes sont basées sur l'analyse d'un corpus de plus de 50 millions de mots publiés en 1998 dans Le Monde et Le Soir, respectivement en France et en Belgique (voir détails dans Verlinde et Selva (2001) et Jones (2001)). On peut ainsi apprendre instantanément quelle proportion d'un texte se retrouve dans les 1000 mots les plus fréquents de la langue française (K1), dans les 1000 mots suivants (K2), dans la troisième tranche (fréquence 2001 à 3000, K3) et dans le groupe 'hors-listes' (MHL), sans parler des informations connexes apportées par plusieurs autres statistiques secondaires. L'intérêt principal de l'exercice est de pouvoir enfin mesurer objectivement la richesse lexicale, qui s'exprime par le nombre de mots 'rares' (fréquence $>2000$ ) trouvés dans un texte. On pourra donc conclure qu'un sujet a fait des 'progrès' lexicaux, 
d'une manière générale, si les scores obtenus dans les tranches de mots 'rares' sont meilleurs dans son posttest que dans son pré-test.

Pour adapter les transcriptions originales des sujets aux exigences de Vocabprofil, il a fallu d'une part corriger l'orthographe pour que l'ordinateur reconnaisse les 'bons' mots et d'autre part éliminer les faux départs, hésitations, etc., ainsi que les noms propres et les mots sémantiquement incorrects, pour qu'il évite de recenser les 'mauvais' mots, attribuant ainsi aux sujets des connaissances lexicales inexistantes. Les pourcentages obtenus au micro-niveau de l'épreuve et de l'individu ont été ensuite regroupés afin d'arriver à des macro-données utilisables pour chaque sujet, chaque épreuve, chaque groupe et l'ensemble des participants. Dans la partie qualitative de l'analyse, nous avons d'abord noté les vocables non-standard trouvés dans le corpus. Nous avons également relevé et analysé les erreurs lexicales commises par les étudiants dans les trois épreuves, considérant comme 'fautive' la non-réalisation ou la réalisation franchement erronée des variantes attendues en français (standard ou québécois), alors que les platitudes ou simples maladresses stylistiques étaient encore traitées comme 'correctes'.

\section{Résultats}

\subsection{Analyse quantitative}

Tableau 1. Mesure de la richesse lexicale en FL1 et FL2 à l'aide de Vocabprofil

\begin{tabular}{|c|c|c|c|c|c|c|c|c|}
\hline \multirow{3}{*}{$\begin{array}{l}\text { Corpus oral de } \\
\text { référence } \\
\begin{array}{l}\text { Notre corpus } \\
\text { expérimental }\end{array}\end{array}$} & K1 & $\mathrm{K} 2$ & K3 & MHL & \multicolumn{3}{|c|}{$\begin{array}{l}\text { K3+MHL Diversité Densité } \\
\text { (image) }\end{array}$} & Mots \\
\hline & & & & & & & & \\
\hline & & & & & & & & \\
\hline pré-test & 88,03 & 3,05 & 0,94 & 6,95 & $7,89(8,49)$ & 0,39 & 0,39 & 45715 \\
\hline post-test & 88,35 & 3,35 & 0,93 & 7,36 & $8,29(8,68)$ & 0,38 & 0,39 & 51085 \\
\hline témoin & & & & & & & & \\
\hline pré-test & 88,48 & 3,45 & 1,12 & 6,93 & $8,05(9,22)$ & 0,38 & 0,38 & 37137 \\
\hline post-test & 89,05 & 3,10 & 1,17 & 6,68 & $7,85(9,03)$ & 0,39 & 0,38 & 37789 \\
\hline cumulatif & 88,48 & 3,24 & 1,04 & 6,98 & $8,02(8,86)$ & 0,39 & 0,39 & 171726 \\
\hline Ovtcharov et al & & & & & & & & \\
\hline B1-faible & 89,18 & 6,05 & 0,77 & 4,02 & 4,79 & & & \\
\hline B2-fort & 87,51 & 6,28 & 1,19 & 5,00 & 6,19 & & & \\
\hline C3-faible & 85,07 & 7,04 & 1,41 & 6,48 & 7,89 & & & \\
\hline
\end{tabular}




\begin{tabular}{|c|cccc|c|}
\hline C4-fort & 81,57 & 7,60 & 2,12 & 8,71 & 10,83 \\
\hline Beeching (FL1) & & & & & \\
& 83,99 & 3,94 & 1,12 & 10,87 & 12,07 \\
\hline
\end{tabular}

Les données présentées dans le tableau 1 démontrent nettement qu'il n'y a aucune différence sensible entre les résultats du pré-test et du post-test, tant pour le groupe expérimental que pour le groupe témoin, et ceci dans toutes les tranches de vocabulaire examinées. De même, la diversité lexicale (rapport vocables / mots) reste constante, tout comme la densité lexicale (rapport mots à sens plein / mots du texte). Faut-il en conclure que les étudiants n'ont fait aucun progrès pendant leur 3e année de français, ni en Ontario, ni en France?

Avant de répondre à cette question, vérifions d'abord la validité pour notre corpus du logiciel Vocabprofil, qui s'est avéré si utile chez nos prédécesseurs, notamment Ovtcharov et al, dont nous reproduisons les résultats dans le tableau 1 à des fins comparatives. Pour ce faire, examinons les résultats partiels obtenus pour les mots dépassant la fréquence 2000 dans l'épreuve de description d'une image complexe par rapport aux parties autobiographique et promotionnelle du test. Dans ces dernières, on s'attend à trouver un texte 'normal', caractérisé par un vocabulaire simple et familier, au choix de l'étudiant-e. Par contre, dans l'image, le vocabulaire est largement imposé par l'image et il est truffé de mots rares (lézard, conducteur, boîte-àsurprises, etc.) apparaissant dans des structures élémentaires du genre "A gauche, il y a un X qui Y une Z". Statistiquement, cela devrait en principe mener à un plus faible pourcentage de vocables communs (puisqu'ils sont constamment répétés) et à un plus grand pourcentage de vocables rares que dans les autres épreuves. Cette différence est effectivement reflétée dans les fréquences $>2000$ (colonne K3+MHL), où les résultats pour l'image seule, présentés entre parentheses, sont constamment plus élevés que pour la moyenne des trois épreuves. Cela signifie qu'il sont encore plus élevés - et de manière significative - par rapport aux pourcentages correspondant séparément à l'autobiographie ou à la promotion de l'université. Voilà donc une différence prévisible qui est dûment mesurée par Vocabprofil, ce qui confirme la validité de cet instrument, déjà établie par nos prédécesseurs, comme on peut le voir dans les résultats progressifs obtenus par Ovtcharov et al (2006). Vocabprofil est également validé par le pourcentage cumulatif de mots rares dans notre corpus $(8,02 \%>2000)$, qui place nos étudiants clairement au-dessus des sujets faibles de la fonction publique $(4,79 \%-6,19 \%)$, ce qui est plausible, et nettement en-dessous des francophones (12,07\%, d'après Beeching, 2001), ce qui l'est encore plus.

S'il est vrai que Vocabprofil est fiable, il faut se demander pourquoi il n'y a aucun progrès apparent dans la richesse lexicale de nos sujets, après une année d'étude en français, surtout en France. Notons tout d'abord qu'il ne faut pas s'attendre à de gros progrès quantitatifs sur une année de la part d'étudiants déjà avancés, contrairement au sujets de Goodfellow et Lamy (2002) et de Milton et Meara (1995), qui étaient de niveau débutant ou intermédiaire. Même dans l'enquête d'Ovtcharov et al (2006), on n'examine les sujets avancés que par rapport à des groupes plus faibles et non entre eux, comme nous le faisons ici. Nous montrerons plus loin qu'il y a des différences sensibles dans notre corpus au niveau qualitatif, mais elles ne sont pas enregistrées par Vocabprofil, qui ne voit aucune différence entre mec, type et individu - tous trois étant dans la catégorie 'hors liste' (MHL) - alors que le choix de l'un ou l'autre de ces vocables en dit long sur les rapports entre le sujet et son contexte d'apprentissage.

Il faut noter également des divergences importantes dans la manière d'évaluer les sujets. Chez Ovtcharov et al (2006: 111), il y a un rapport étroit entre les cours de terminologie professionnelle et l'évaluation subséquente, pendant laquelle on pose des questions "sur l'environnement du travail, les tâches, les problèmes et le cheminement de carrière des candidats, et les civilités quotidiennes au début et à la fin de l'entrevue". On ne sera donc pas surpris de constater (tableau 1) que le test mesure effectivement les acquisitions recherchées. 
Par contraste, nos étudiants ont été testés sur des connaissances spécifiques (l'image) ou générales (l'autobiographie et le message promotionnel), qui n'avaient aucun rapport direct avec leur curriculum ou leurs contacts avec les milieux francophones. Qui plus est, les deux dernières épreuves étaient suffisamment élémentaires pour ne pas requérir le genre de sophistication nécessaire à la description d'une image complexe ou du milieu professionnel des fonctionnaires. Les sujets pouvaient fort bien se tirer d'affaire avec un niveau 2e année et il n'est pas évident que leurs acquisitions subséquentes leur aient beaucoup servi dans les tâches

les plus faciles de notre test. On ne voit pas bien non plus les différences entre une Ferrari et une petite Renault en se rendant simplement à l'épicerie du coin...

Force est donc de constater que les acquisitions lexicales probables de nos étudiants avancés au niveau passif - malheureusement exclues de notre analyse - ne se traduisent pas nécessairement par des progrès quantitatifs sur le plan actif. Il faudra donc entreprendre une analyse qualitative (voir plus bas) pour découvrir ce qui fait la spécificité du séjour à l'étranger sur le plan lexical au niveau avancé.

Finalement, la seule différence quantitative notable entre les deux groupes se situe au niveau du nombre de mots utilisés pendant les 13 minutes de test. On peut voir, dans la colonne "Mots" du tableau 1, que le groupe expérimental augmente sa production de manière significative $(11.7 \%)$, alors que le groupe témoin se contente d'une augmentation marginale de $1.7 \%$. Ceci confirme l'analyse du même corpus du point de vue de la vitesse d'élocution, qui conclut que l'aisance acquise en France se traduit par une plus grande réduction dans le temps de planification, les hésitations et autres dysfonctions de l'oral. Les sujets de notre groupe expérimental n'ont peut-être pas fait de progrès en richesse lexicale, du moins au niveau de la production orale, mais ils ont certainement acquis une plus grande aisance dans l'expression de leur pensée. C'est peutêtre là que se trouve l'acquis linguistique le plus important de l'année à l'étranger.

\subsection{Analyse qualitative}

\subsubsection{Vocabulaire non-standard}

On trouvera ci-dessous la liste exhaustive des vocables non-standards relevés dans notre corpus, avec entre parenthèses le nombre de sujets chez qui le mot en question est apparu au moins une fois. En principe, ces vocables n'apparaissent pas en langue écrite, ni en langue orale soignée, qui constituent en gros les limites de notre enseignement du français dans les universités de l'Ontario. Leur utilisation reflète un contact plus ou moins étroit avec le français oral familier, qui est prédominant pendant le séjour à l'étranger. C'est donc dans le groupe expérimental qu'on peut s'attendre à trouver une plus forte utilisation du français non-standard.

Abbréviations:

prof (x29), sympa (x25), fac (x6), resto (x2), basket (x2), boîte (x2), télé, tram, pull, foot, frigo, maths, ciné, coloc

Termes populaires, argotiques:

mec (x6), balade / se balader (x5), copain / copine ( 55$)$, flic ( $x 4)$, bonhomme ( $x 4)$, relax (x3), salut (x2), marrant (x2), chouette, cafard, black, déglingué, nounours, pas pire, type, bonne femme, boire un coup, bouquin, gars, gamine, branché, boulot, rigolo, ça pue, dégueulasse

\section{Exclamations et remplisseurs:}

génial (x11), truc (x11), super (x4), ben (x3), quoi (x2), machin (x2), hyper, vachement, pas mal de, hop, bordel, fait chier 
TOTAL Groupe expérimental: 38 vocables (pré-test) $\rightarrow 115$ (post-test)

Groupe témoin: $\quad 33$ vocables (pré-test) $\rightarrow 25$ (post-test)

Si l'on compare le nombre total des productions non-standards pour les deux groupes et les deux périodes de test - malgré les dangers d'une telle entreprise (par exemple, on ne tient pas compte de la taille du corpus de référence) - on constate immédiatement que ces productions ont triplé pendant le séjour à l'étranger, alors qu'elles ont diminué chez les sujets restés en Ontario pendant la même période. Sur le plan qualitatif, hormis deux étudiants francophones, ces derniers se limitent généralement à trouver leurs profs sympas. Le reste de la liste ci-dessus se trouve presque entièrement dans le corpus expérimental. Apparemment donc, c'est surtout en séjournant en milieu francophone qu'on apprend à tronquer les mots, à parler familièrement ou vulgairement, à s'exclamer comme les natifs et à utiliser les mots passe-partout du genre truc ou machin qui évitent les pannes de communication. C'est manifestement en fréquentant la rue qu'on 'attrape' le français de la rue, ce qui ne surprendra personne.

\subsubsection{Erreurs lexicales}

Partant de l'hypothèse qu'une année passée à étudier et pratiquer le français devrait améliorer la qualité de l'expression lexicale, on a recensé les erreurs de vocabulaire commises dans les tests, le terme 'erreur' étant défini pragmatiquement comme toute forme ou sens carrément inacceptable pour l'enquêteur, qui est un francophone expérimenté en français standard et québécois, les deux seuls dialectes du français véritablement présents dans l'expérience des sujets. Voici la liste des erreurs fréquentes, c'est-à-dire celles qu'on a relevées chez au moins 5 sujets:

\section{$\underline{\text { Faux-amis }}$}

un signe (un signal, un panneau, une pancarte), chasser (poursuivre), pour (pendant), le bol (le bocal), pointer (indiquer), être $x$ ans (avoir), marcher son chien (promener), jouer des sports (pratiquer), prendre le français (étudier), prendre quelqu'un (emmener), joindre un club (adhérer, s'inscrire), les courses (les cours), regarder comme (sembler), le couvert (la couverture), les lumières de trafic (feux de circulation), une part (partie), la loi (le droit), croiser (barrer), la copie (l'exemplaire), les temps (une période)

\section{Mots anglais}

le pole, le post (poteau), la crane (la grue), le jack-in-the-box (la boîte-à-surprise), le pail, le bucket (le seau), un cell phone (un cellulaire), une rocket (une fusée), une arrow (une flèche), en kindergarten (en maternelle), upside down (à l'envers)

\section{$\underline{\text { Autres }}$}

le magasin (le magazine), un gens (une personne), une madame / mademoiselle (dame / demoiselle), un conduiseur (un conducteur), un demi (la moitié), savoir (connaître), mal (mauvais), beaucoup (très) 
Tableau 2. Erreurs lexicales (vocables) relevées dans le corpus

\begin{tabular}{|l|lrccc|}
\hline Référence & \multicolumn{5}{|c|}{ Erreurs lexicales (vocables) } \\
& Faux-amis Mots angl. Autres TOTAL Erreurs/sujet \\
\hline $\begin{array}{l}\text { Groupe expérim. } \\
\text { pré-test }\end{array}$ & 210 & 81 & 104 & 395 & 8,2 \\
post-test & 119 & 40 & 79 & 238 & 5 \\
$\begin{array}{l}\text { Groupe témoin } \\
\text { pré-test }\end{array}$ & 254 & 133 & 104 & 491 & 12,6 \\
post-test & 198 & 87 & 90 & 375 & 9,6 \\
& 781 & 341 & 377 & 1499 & 17,2 \\
\hline
\end{tabular}

Le tableau 2, qui souffre des mêmes défauts que les données sur le français non-standard, permet tout de même de faire plusieurs observations utiles sur la qualité du vocabulaire de nos étudiants avancés. Globalement, un total de 1499 erreurs sur un ensemble de 66973 vocables (nombre de mots x densité lexicale, mentionnés dans le tableau 1) donne un taux d'erreur de l'ordre de $2 \%$, qui paraît minime a priori. Cela confirme l'impression de nos assistants francophones, qui n'ont pas eu beaucoup de mal à transcrire les enregistrements des étudiants, bien qu'une certaine connaissance de l'anglais ait souvent facilité les choses (voir ci-dessous). Il s'agit après tout du FL2 avancé.

Sur le plan de la distinction entre les groupes, on remarque que tout le monde fait moins d'erreurs en fin d'année, dans toutes les catégories - ce qui est rassurant... - et que les sujets du groupe expérimental progressent un peu plus que les autres, sans que ce soit statistiquement évident. Ce qui est plus clair, par contre, c'est que la grande majorité des erreurs, environ 75\%, provient de l'interférence de l'anglais, L1 de la plupart des sujets. De plus, dans ce dernier groupe, ce sont les faux-amis qui dominent - par rapport aux mots donnés carrément en anglais - c'est-à-dire les erreurs les moins visibles et donc les moins faciles à corriger. On peut longtemps dire chasser (pour poursuivre) sans risquer d'être mal compris et en ayant l'impression de ne parler rien d'autre que le français.

Enfin, sur le plan strictement qualitatif, la liste révèle des erreurs prévisibles, comme bol pour bocal, mais aussi des erreurs fondamentales, qu'on croyait avoir éliminées depuis longtemps et qui sont rarement corrigées entre le pré-test et le post-test. Pourquoi, se demande-t-on, trouve-t-on encore en troisième année d'université des étudiants qui sont vingt ans, ont pris le français dans des courses spécialisés pour une dizaine d'années? Ca regarde comme si ces apprenants ont manqué une partie essentielle de leur formation et que nous sommes ici en présence de fossilisation lexicale. Il semblerait que certaines erreurs s'incrustent dans le français de nos étudiants et que même une année en France n'arrive pas à les déloger. 


\section{Conclusion}

L'analyse d'un corpus de FL2 avancé a permis de tester la validité du logiciel Vocabprofil comme mesure de la richesse lexicale en français et de constater qu'il est surtout utile pour mesurer les grandes différences de niveau et quand il y a symbiose entre la matière enseignée et la matière testée. Nos étudiants ont sûrement fait des progrès en vocabulaire, du moins au niveau passif et surtout s'ils viennent de passer une année en France,

mais ces progrès ne se traduisent pas nécessairement par des augmentations mesurables à l'aide de Vocabprofil dans un test général de production verbale. Le problème est accentué par le fait qu'il s'agit ici d'étudiants avancés, qui étaient déjà capables d'accomplir convenablement les tâches exigées avant même de commencer leurs études de troisième année. Le test choisi ici, pourtant adéquat pour étudier la prononciation et la grammaire des étudiants, s'avère donc insuffisant pour analyser leur vocabulaire. Seul le calcul du nombre de mots utilisés en un certain laps de temps permet de confirmer les résultats d'une étude antérieure sur la facilité d'élocution. Il est clair que le groupe expérimental est beaucoup plus à l'aise dans son expression orale à l'issue du séjour en France que le groupe témoin après plusieurs cours de français en Ontario. C'est probablement là, d'après l'ensemble de nos recherches sur le sujet, la principale vertu linguistique du séjour à l'étranger.

L'analyse qualitative du corpus a révélé que les erreurs commises sur le plan lexical n'entravent généralement pas la communication au niveau avancé et qu'on en réduit le nombre en passant une année à étudier le français, surtout si on le fait en milieu francophone. Mais certains anglicismes - de loin la plus importante cause d'erreurs - sont particulièrement tenaces et il est clair qu'on ne peut pas compter sur l'immersion totale pour éliminer les erreurs qui sont déjà fossilisées. Ceci devrait encourager les enseignants aux niveaux inférieurs du système éducatif à accorder plus d'importance à la forme - sans toutefois sacrifier la communication - sous peine de créer un franglais qui sera difficile à déloger par la suite.

Enfin, la plus saillante des différences entre les deux groupes révélées par notre analyse est sans conteste l'utilisation relative du français non-standard. Les sujets ayant séjourné en France reviennent avec un grand nombre de mots familiers ou argotiques - voire vulgaires - inconnus de leurs camarades restés en Ontario, preuve qu'ils ont particulièrement bien absorbé le lexique de la rue dans lequel ils viennent de naviguer pendant une année. Mais cette acquisition est un couteau à double tranchant, comme on peu le voir dans cet extrait de la description d'image produite par l'une de nos étudiantes après une année passée à Nice: "Un mec... ben, sa tête n'est pas attachée. Ce mec a un mec dans son poche et ses pieds sont pas attachés... son jambe. Et un mec avec un queue. Un squelette qui vend des journaux, avec un mec en haut". Dans un sens, cette étudiante a fait des progrès, puisque son lexique s'est rappoché de la L1 telle qu'elle est pratiquée par ses homologues français $(\mathrm{mec}$ ). Mais ces progrès s'inscrivent dans une morpho-syntaxe défectueuse, surtout marquée par de grossières erreurs de genre et un ne formel qui contraste avec la familiarité du lexique. La question se pose alors de savoir si l'acquisition du vocabulaire familier est une bonne chose quand elle n'est pas accompagnée d'acquisitions similaires dans les autres domaines de la langue. Faut-il mieux dans ce cas se limiter à l'enseignement du français standard, qui aura au moins le mérite d'être cohérent dans son imperfection?

\section{Références bibliographiques}

Beeching, K. (2001). Un corpus d'entretiens spontanés, enregistrés et transcrits par Kate Beeching. [En ligne] Disponible sur http://www.uwe.ac.uk/facults/les/staff/kb/CORPUS.pdf

Churchill, E. et Dufon, M.A. (2006). Evolving threads in study abroad research. In Churchill, E. et Dufon,

M.A. (eds), Language Learners in Study Abroad Contexts. Ch. 1. Clevedon: Multilingual Matters.

Cobb, T. (2003). Analyzing late interlanguage with learner corpora. Quebec replications of three European 
studies. Canadian Modern Language Review, 59(3), 393-423.

Cobb, T. et Horst, M. (2004). Is there room for an academic word list in French? In P.Bogaards et Laufer,

B. (eds), Vocabulary in Second Language. Amsterdam: Benjamins, 15-38.

Collentine, J. (2004). The effects of learning contexts on morphosyntactic and lexical development. Studies in SecondLanguage Acquisition, 26 (2), 227-248.

Dewaele, J.-M., (2004). Individual differences in the use of colloquial vocabulary: the effects of sociobiographical and psychological factors. In P. Boogaards et B. Laufer (eds), Vocabulary in Second Language. Amsterdam: Benjamins, 126-153.

Dewaele, J.-M. et Pavlenko, A. (2002). Emotion vocabulary in interlangage, Language Learning, 52 (2), 263-322.

Freed, B. F. (1995). Language learning and study abroad. In B.F. Freed (ed.) Second-Language Acquisition in a StudyAbroad Context. Amsterdam: Benjamins, p. 3-33.

Goodfellow, R. et Lamy, M.-N. (2002). Assessing learners' text using the Lexical Frequency Profile. ReCALL, 14(1), $133-145$.

Jones, G. (2001). Compiling French word frequency lists for the Vocabulary Assessment Tool: A Feasibility Study. [En ligne] Disponible sur http://www.er.uqam.ca/nobel/r21270/cgi-bin/F_webfreqs/glynn jones.html

Laniel, D. (2005). Le vocabulaire en français langue seconde: de 'parent pauvre' de l'enseignement à 'invité d'honneur' des communications assistées par ordinateur. Revue de l'AQEFLS, 25 (2), 73-100.

Laufer, B. (1986). Possible changes in attitude toward vocabulary acquisition research. IRAL, 24(1), 69-76.

Laufer, B. et Nation, P. (1995). Vocabulary size and use: Lexical richness in L2 written Production. Applied Linguistics, $16(3), 307-322$

Meara, P. (1984). The study of lexis in interlanguage. In Davis, A., C. Criper, Howatt, A.P.R. (eds.), Interlanguage. Edinburgh: Edinburgh U. Press, 225-235.

Milton, J. et Meara, P. (1995). How periods abroad affect vocabulary growth in a foreign Language. ITL Review of Applied Linguistics, 107/108: 17-34.

Muller, C. (1979). Langue française et linguistique quantative. Genève: Slatkine.

Ovtcharov, V., Cobb, T. et Halter, R.. (2006). La richesse lexicale des productions orales:mesure fiable du niveau de compétence langagière. Canadian Modern Language Review, 63(1), 107-125.

Tréville, M.-C. et Duquette, L. (1996). Enseigner le vocabulaire en classe de langue. Paris: Hachette.

Velinde, S. et Selva, T. (2001). Corpus-based versus intuition-based lexicography: Defining a word list for a French learners' dictionary. [En ligne] Disponible sur http://www.kuleuven.ac.be/ilt/grelep/publicat/verlinde.pdf 